\title{
In-hospital heart rate reduction and its relation to outcomes of heart failure patients with sinus rhythm: Results from the Polish part of the European Society of Cardiology Heart Failure Pilot and Long-Term Registries
}

Paweł Balsam ${ }^{1}$, Michał Peller ${ }^{1}$, Sonia Borodzicz ${ }^{1}$, Agnieszka Kapłon-Cieślicka ${ }^{1}$, Krzysztof Ozierański ${ }^{1}$, Agata Tymińska ${ }^{1}$, Michał Marchel ${ }^{1}$, Maria G. Crespo-Leiro ${ }^{2}$, Aldo Pietro Maggioni ${ }^{3,4}$, Jarosław Drożd $\dot{z}^{5}$, Marcin Grabowski ${ }^{1}$, Krzysztof J. Filipiak ${ }^{1}$, Grzegorz Opolski ${ }^{1}$

${ }^{1} 1^{\text {st }}$ Chair and Department of Cardiology, Medical University of Warsaw, Poland

${ }^{2}$ Unidad de Insuficiencia Cardiaca Avanzada y Trasplante Cardiaco,

Hospital Universitario A Coruna, CIBERCV, La Coruna, Spain

${ }^{3}$ ANMCO Research Center, Florence, Italy

${ }^{4}$ EURObservational Research Programme, European Society of Cardiology, Sophia-Antipolis, France

${ }^{5}$ Department of Cardiology, Medical University, Lodz, Poland

\begin{abstract}
Background: Currently, there is no information on whether in-hospital heart rate (HR) reduction has an influence on risk of death or rehospitalization. The study evaluates the relation between inhospital HR reduction in heart failure (HF) patients on mortality and rehospitalization within 1-year observation.

Methods: The analysis included patients hospitalized in Poland with sinus rhythm from the European Society of Cardiology Heart Failure Pilot (ESC-HF-Pilot) and ESC Heart Failure Long-Term Registries (ESC-HF-LT), who were divided into two groups: reduced HR and not-reduced HR. HR reduction was defined as a reduced value of HR at discharge compared to admission HR. The primary endpoint was 1-year all-cause death, the secondary endpoint was 1-year all-cause death or rehospitalization for worsening $H F$.
\end{abstract}

Results: The final analysis included 747 patients; 491 reduced HR (65.7\%) and 256 not-reduced HR (34.3\%). The primary endpoint occurred in 58/476 (12.2\%) from reduced HR group and in 26/246 (10.5\%) from not-reduced HR group $(p=0.54)$. In the reduced HR group, independent predictors of primary endpoint were age, New York Heart Association class at admission, serum sodium level at admission and systolic blood pressure at discharge. In the not-reduced HR group the independent predictor of primary endpoint was diastolic blood pressure at discharge. The secondary endpoint was observed in 180 patients, 124/398 (31.2\%) from reduced HR and 56/207 (27.1\%) from the not-reduced HR group $(p=0.30)$. In the not-reduced HR group only angiotensin converting-enzyme inhibitor usage at discharge was independently associated with lower risk of the secondary endpoint.

Conclusions: In-hospital HR reduction did not influence on the outcomes of HF patients in sinus rhythm. (Cardiol J 2020; 27, 1: 25-37)

Key words: heart failure, registry, prognosis, heart rate, hospitalization

Address for correspondence: Michał Peller, MD, $1^{\text {st }}$ Chair and Department of Cardiology, Medical University of Warsaw, Public Central Teaching Hospital in Warsaw, ul. Banacha 1a, 02-097 Warszawa, Poland, tel: +48 22 599-19-58,

fax: +48 22 599-19-57, e-mail: michalpeller@gmail.com

Received: 5.02.2018

Accepted: 7.07.2018 


\section{Introduction}

Although the treatment of heart failure (HF) has been improving in recent decades, the outcome of $\mathrm{HF}$ patients is still not satisfactory $[1,2]$. Increasing prevalence of $\mathrm{HF}$ in developing countries is a great challenge for contemporary cardiology. Proper identification of risk factors of death or rehospitalization is crucial for the management of HF patients.

The most comprehensive and reliable data concerning the risk factors and outcome of patients with HF come from international observation registries. The European Society of Cardiology (ESC) created the Heart Failure Pilot (ESC-HF-Pilot) and Heart Failure Long-Term (ESC-HF-LT) Registries to assess the clinical characteristics and outcome of HF patients in clinical practice in European countries. Recently published analyses of data from both Registries revealed several risk factors associated with 1-year outcomes in hospitalized HF patients [1,3-6]. One of the modifiable predictors of cardiovascular mortality and morbidity is heart rate (HR), which is associated with poor prognosis in general population, patients with hypertension, coronary artery disease and HF [2, 7-11]. Laskey et al. [12] reported, that higher $\mathrm{HR}$ at discharge in hospitalized HF patients significantly increased the risk of death or rehospitalization. However, there is still no information on whether in-hospital reduction of HR modifies risk of death or rehospitalization.

The aim of the current analysis was to evaluate the influence of in-hospital $\mathrm{HR}$ reduction in $\mathrm{HF}$ patients with sinus rhythm (SR) on mortality and/or rehospitalization over a 1-year observation period.

\section{Methods}

\section{Study population}

In the present analysis, data from two prospective, multicenter registries were included: ESC-HF-Pilot and ESC-HF-LT [1, 2, 13, 14]. The ESC-HF-Pilot Registry included data gathered between October 2009 and May 2010 in 136 European centers, including 29 centers localized in Poland. The ESC-HF-LT Registry consists of three phases, including data from 211 centers in 21 European countries. The I phase of the ESC-HF-LT Registry was conducted between May 2011 and April 2013 and enrolled patients 1 day per week for the whole year. Adult patients (at least 18 years old) with newly-diagnosed HF (using clinical, biochemical and echocardiographic findings) or worsening of $\mathrm{HF}$ were enrolled in the Registries. The ESC-HF-Pilot and ESC-HF-LT Registries recruited patients hospitalized for $\mathrm{HF}$ and outpatients seen in ambulatory care. Exclusion criteria were not specified. All patients signed an informed consent. The study was approved by the local Ethical Review Board.

In the current analysis only hospitalized patients enrolled in the ESC-HF-Pilot Registry and in phase I of the ESC-HF-LT Registry in SR were taken into account. Atrial fibrillation/atrial flutter and/or paced rhythm on 12-lead electrocardiogram (ECG), as well as lack of ECG recording during index hospitalization were excluded from the current analysis.

All data according to the medical history, concomitant diseases and clinical status at admission and hospital discharge were obtained. Follow-up of the patients lasted 1 year. During the follow-up data regarding all-cause death and readmission for HF worsening were collected.

\section{Study groups}

Patients were divided into two groups according to HR difference during index hospitalization from admission to discharge: with or without HR reduction. HR values were assessed during standard physical examination. HR reduction was defined as a reduced value of HR recorded at discharge in comparison to the value observed upon admission. Patients with HR reduction (reducedHR group) and without HR reduction (not-reducedHR group) during index hospitalization were compared in regard to demographics, medical history, clinical status and pharmacotherapy at the moment of admission, during index hospitalization and at hospital discharge.

\section{Endpoints}

In both Registries, the primary endpoint was 1-year all-cause death, whereas the secondary endpoint was composed of 1-year all-cause death or rehospitalization for worsening HF.

\section{Statistical analysis}

Normality of distribution of variables was assessed using the Shapiro-Wilk test. Continuous non-normally distributed variables were presented as median values and interquartile range (IQR). Categorical data were presented as percentage and absolute frequencies. Statistical significance of differences between groups was assessed: for quantitative variables with U Mann-Whitney test and for qualitative variables - with Fisher exact test. Cox proportional hazard regression models were used to determine predictors of the primary 


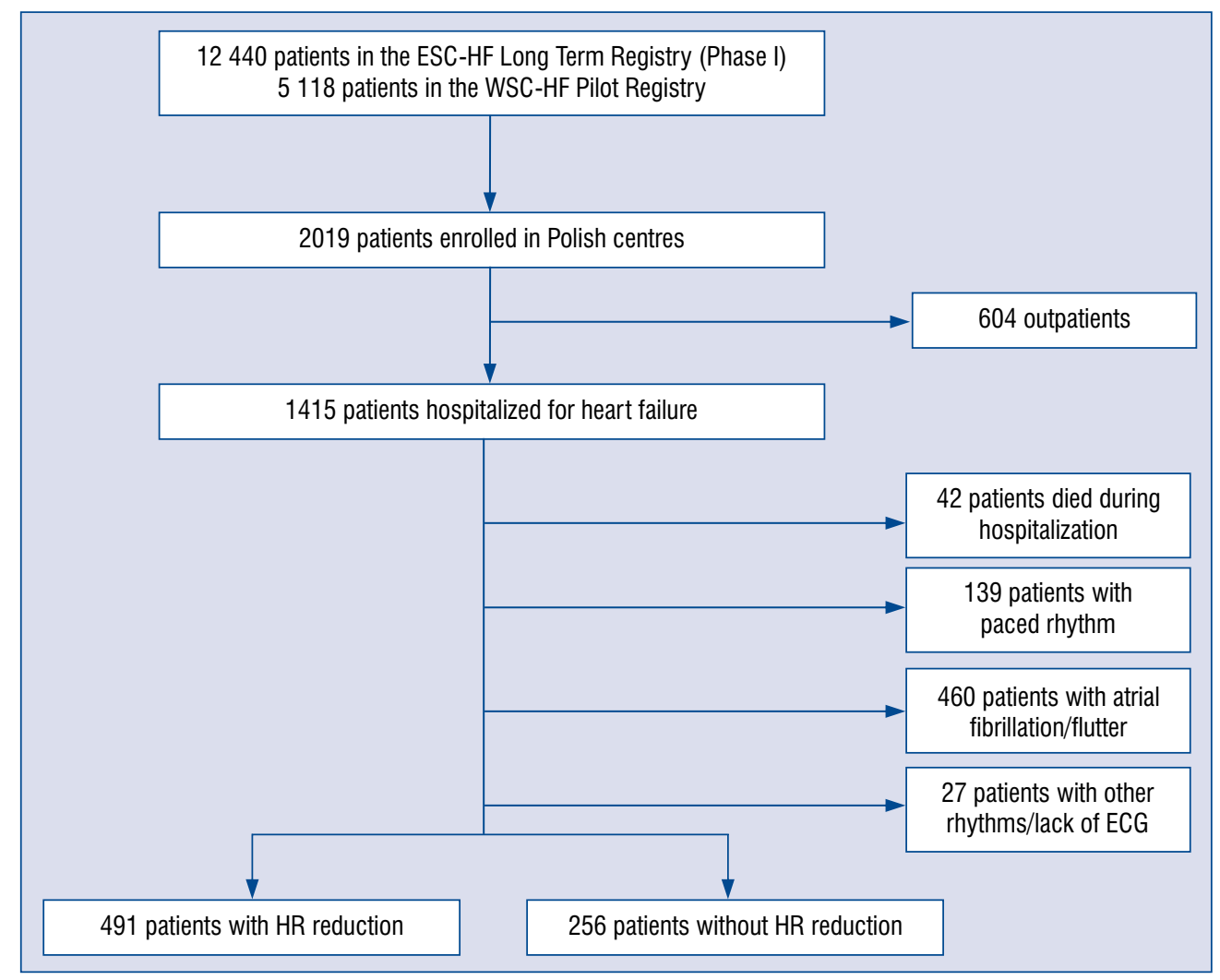

Figure 1. The flow chart of patient recruitment in the study; HR — heart rate; ECG - electrocardiogram.

and secondary endpoints. Only variables with $p<0.1$ in univariate analysis were included in multivariate analysis. A value of $p<0.05$ was considered significant for all tests. Statistical analysis performed using $\mathrm{SAS}^{\circledast}$ software, version 9.4.

\section{Study group selection}

Overall, in all European countries 5118 patients were enrolled in the ESC-HF-Pilot Registry and 12,440 patients in the ESC-HF-LT Registry. The Polish cohorts of the ESC-HF-Pilot and ESCHF-LT Registries included 630 and $743 \mathrm{HF}$ patients who were discharged after index hospitalization, respectively. Firstly, 139 patients were excluded from the current analysis, because of paced rhythm observed in ECG recording. Secondly, 460 patients with present atrial fibrillation/atrial flutter during index hospitalization were excluded from further analysis. Other rhythms or lack of ECG were noted in 27 patients. Finally, ECG recordings on admission and discharge were available for 747 (100\%) patients. The flow chart of patient enrollment in the analysis is shown in Figure 1. HR reduction was observed in 491 of 747 (65.7\%) patients, while lack of HR reduction in 256/747 (34.3\%) patients included in the study. Follow-up data was excluded for 25 patients, resulting from a lack of response after direct, investigator contact.

\section{Results}

\section{Study group characteristics}

Median age in the group analyzed was 67.0 (57.6-77.0) years, $68.5 \%$ of patients were male. Median HR value at admission in the total population was $80(70-90)$ beats per minute (bpm). In the reduced $\mathrm{HR}$ group median $\mathrm{HR}$ at admission was 84 (75-100) bpm, whereas in the not-reduced HR group $70(60-75)$ bpm $(\mathrm{p}<0.0001)$. Furthermore, median HR value at discharge was 70 (64-78) bpm in the population analyzed, $70(62-75) \mathrm{bpm}$ in the reduced HR group and $72(68-80) \mathrm{bpm}$ in the notreduced HR group $(\mathrm{p}<0.0001)$. Median value of $\mathrm{HR}$ reduction in the reduced HR group was $15 \mathrm{bpm}$ (IQR: 8-25 bpm). The reduced HR group more frequently had hypertension $(71.0 \%$ vs. $63.3 \%$; $\mathrm{p}=0.04)$ and less frequently used antiplatelets before the index hospitalization (58.2\% vs. $69.4 \%$; $\mathrm{p}=0.003)$ in comparison to the not-reduced HR group. According to clinical status at admission, 
the reduced HR group had higher New York Heart Association (NYHA) class (3 [2-4] vs. 3 [2-3]; $\mathrm{p}=0.02$ ), higher systolic blood pressure (SBP) (131 [120-150] vs. 130 [110-140]; $p=0.002)$, higher diastolic blood pressure (DBP) (80 [70-90] vs. 80 [70-84]; $\mathrm{p}=0.0005$ ) and more frequently were admitted because of acute coronary syndrome ([ACS] $27.5 \%$ vs. $20.7 \%$; $=0.04$ ). Moreover, reduced HR group had a longer duration of index hospitalization (7 [4-11] vs. 6 [3-9]; $\mathrm{p}=0.004$ ), in comparison to the not-reduced HR group. A full comparison of both groups in regard to baseline characteristics, clinical course of index hospitalization, in-hospital and long-term outcomes are presented in Table 1. As shown in Table 2, HR at admission was significantly higher in the ESC-HF-Pilot Registry population in comparison to the group enrolled in the ESC-HF-LT Registry (80 [70-95] vs. 78 [68-90]; $p=0.02$ ). The comparison between these two Registries did not show significant differences in regard to HR at discharge, mean HR reduction during hospitalization or the percentage of patients who achieved HR reduction (Table 2).

\section{One-year outcomes}

Moreover, no significant differences were observed between groups in occurrence of primary and secondary endpoints. In comparison of reduced HR and not-reduced HR groups, hazard ratios of prevalence of primary and secondary endpoints were 1.16 (95\% confidence interval [CI] 0.73-1.84; $\mathrm{p}=0.54)$ and $1.15(95 \%$ CI $0.85-1.56 ; \mathrm{p}=0.38)$, respectively. Kaplan-Meier curves present outcomes of reduced HR and not-reduced HR groups are shown in Figure 2.

\section{Primary endpoint}

In the population analyzed, 722 patients completed 1-year follow-up and primary endpoint occurred in 84/722 patients (11.6\%). In the reduced HR group, primary endpoint was observed more frequently $(58 / 476,12.2 \%)$, than in the not-reduced HR group $(26 / 246,10.5 \%$; $=0.54)$. Tables 3 and 4 present risk factors for 1-year all-cause death in univariate analysis in the reduced HR and not-reduced HR groups, respectively. In the multivariate analysis only older age, higher NYHA class at admission, lower serum sodium at admission and lower SBP at discharge were revealed to be independent predictors of primary endpoint in the reduced HR group (Table 5). In multivariate analysis only lower DBP at discharge remained to be a statistically significant predictor of 1 -year all-cause death in the not-reduced HR group, as shown in Table 6.

\section{Secondary endpoint}

In the total population, data on 1-year followup were available for 605 patients. In the whole analyzed group, secondary endpoint was observed in $180(29.8 \%)$ patients, $124 / 398(31.2 \%)$ from the reduced HR and 56/207 (27.1\%) from the not-reduced HR group $(\mathrm{p}=0.30)$. Tables 3 and 4 present risk factors for secondary endpoint in univariate analysis in the reduced HR and not-reduced HR groups, respectively. In the reduced HR group, the multivariate analysis did reveal these factors to reach statistical significance (Table 5). However, there were trends for diabetes, history of stroke, higher NYHA class at admission and lower serum sodium at admission towards independent prediction of secondary endpoint in the reduced HR group. In the not-reduced HR only the use of angiotensin converting enzyme inhibitor at discharge was independently associated with lower risk of all-cause death or rehospitalization for worsening $\mathrm{HF}$, as presented in Table 6 .

\section{Discussion}

The current study has revealed that HR reduction during the hospitalization for HF was not associated with benefits in patients with SR. Moreover, predictors of all-cause death or combined endpoint (death or rehospitalization for worsening HF) at 1 year were partly comparable in patents with and without HR reduction during index hospitalization.

Among numerous demographic and clinical factors, only a few of them differed between patients with and without in-hospital HR reduction. In the reduced-HR group higher NYHA class was observed. Not much is known about the correlation between NYHA class and HR at hospital admission. However, Ahmed et al. [15] revealed no significant differences in $\mathrm{HR}$ at admission and NYHA class I-II vs. III-IV in patients with HF with preserved function of the left ventricle. Moreover, results from the current analysis showed that in the reduced HR group, higher NYHA class at admission is significantly related to all-cause death at 1 year. These findings are consistent with results of previous analyses performed in hospitalized $\mathrm{HF}$ patients enrolled in the ESC-HF-Pilot and ESC-HF-LT Registries [1, 3, 4].

In the present analysis, the reduced HR group less frequently used beta-blockers (BBs) prior to 
Table 1. Baseline characteristics, clinical course of index hospitalization, in-hospital and long-term outcomes of the reduced HR and not-reduced HR groups.

\begin{tabular}{|c|c|c|c|c|}
\hline & $\begin{array}{c}\text { Total } \\
(\mathrm{n}=747)\end{array}$ & $\begin{array}{l}\text { Not-reduced HR } \\
\quad(n=256)\end{array}$ & $\begin{array}{l}\text { Reduced HR } \\
(n=491)\end{array}$ & $\mathbf{P}$ \\
\hline \multicolumn{5}{|l|}{ Demographics } \\
\hline Age [years] & $\begin{array}{c}67.0(57.6-77.0) \\
n=747\end{array}$ & $\begin{array}{c}67.0(58.0-76.7) \\
n=256\end{array}$ & $\begin{array}{c}67.0(57.6-77.0) \\
n=491\end{array}$ & 0.92 \\
\hline Male & $68.5 \% ; 512 / 747$ & $70.7 \% ; 181 / 256$ & $67.4 \% ; 331 / 491$ & 0.41 \\
\hline BMI $\left[\mathrm{kg} / \mathrm{m}^{2}\right]$ & $\begin{array}{c}27.7(24.7-31.2) \\
n=708\end{array}$ & $\begin{array}{c}27.7(24.9-30.6) \\
n=244\end{array}$ & $\begin{array}{c}27.7(24.5-31.6) \\
n=464\end{array}$ & 0.76 \\
\hline \multicolumn{5}{|l|}{ Heart failure } \\
\hline LVEF [\%] & $35(25-50) ; n=669$ & $37(26-50) ; n=213$ & $35(25-50) ; n=456$ & 0.70 \\
\hline \multicolumn{5}{|l|}{ Medical history } \\
\hline Hypertension & $68.4 \% ; 510 / 746$ & $63.3 \% ; 162 / 256$ & $71.0 \% ; 248 / 490$ & 0.04 \\
\hline Coronary artery disease & $61.5 \% ; 459 / 746$ & $64.5 \% ; 165 / 256$ & $60.0 \% ; 294 / 490$ & 0.33 \\
\hline Peripheral artery disease & $12.5 \% ; 92 / 747$ & $11.3 \% ; 29 / 256$ & $12.8 \% ; 63 / 491$ & 0.64 \\
\hline Diabetes & $33.7 \% ; 252 / 747$ & $33.2 \% ; 85 / 256$ & $34.0 \% ; 167 / 491$ & 0.87 \\
\hline Chronic kidney disease & $18.2 \% ; 136 / 746$ & $17.2 \% ; 44 / 256$ & $18.8 \% ; 92 / 490$ & 0.62 \\
\hline COPD & $16.4 \% ; 122 / 745$ & $12.6 \% ; 32 / 255$ & $18.4 \% ; 90 / 490$ & 0.05 \\
\hline Stroke & $7.8 \% ; 58 / 746$ & $5.5 \% ; 14 / 256$ & $9.0 \% ; 44 / 490$ & 0.11 \\
\hline \multicolumn{5}{|l|}{ Previous pharmacotherapy } \\
\hline Diuretics & $62.2 \% ; 452 / 727$ & $66.3 \% ; 167 / 252$ & $60.0 \% ; 285 / 475$ & 0.11 \\
\hline Aldosterone antagonist & $40.0 \% ; 291 / 727$ & $43.7 \% ; 110 / 252$ & $38.1 \% ; 181 / 475$ & 0.15 \\
\hline ACEI & $62.6 \% ; 455 / 727$ & $65.1 \% ; 164 / 252$ & $61.3 \% ; 291 / 475$ & 0.33 \\
\hline ARB & $9.8 \% ; 71 / 725$ & $8.4 \% ; 21 / 251$ & $10.6 \% ; 50 / 474$ & 0.43 \\
\hline Beta-blocker & $72.6 \% ; 527 / 726$ & $75.4 \% ; 190 / 252$ & $71.1 \% ; 337 / 474$ & 0.22 \\
\hline Statins & $57.2 \% ; 415 / 726$ & $61.5 \% ; 165 / 252$ & $54.9 \% ; 260 / 474$ & 0.10 \\
\hline Ivabradine & $0.3 \% ; 1 / 391$ & $0.0 \% ; 0 / 145$ & $0.4 \% 1 / 246$ & 1.00 \\
\hline Antiplatelets & $62.1 \% ; 451 / 726$ & $69.4 \% ; 175 / 252$ & $58.2 \% ; 276 / 474$ & 0.003 \\
\hline \multicolumn{5}{|l|}{ Clinical status at admission } \\
\hline Cardiogenic shock & $1.8 \% ; 13 / 708$ & $1.3 \% ; 3 / 237$ & $2.1 \% ; 10 / 471$ & 0.56 \\
\hline NYHA class & $3(2-4) ; n=743$ & $3(2-3) ; n=256$ & $3(2-4) ; n=487$ & 0.02 \\
\hline NYHA I & $1.4 \% 10 / 719$ & $1.6 \% 4 / 256$ & $1.3 \% 6 / 487$ & \\
\hline NYHA II & $28.7 \% 206 / 719$ & $31.3 \% 80 / 256$ & $27.5 \% 129 / 487$ & \\
\hline NYHA III & $44.1 \% 317 / 719$ & $48.1 \% 123 / 256$ & $43.1 \% 201 / 487$ & \\
\hline NYHA IV & $35.9 \% 186 / 719$ & $19.1 \% 49 / 256$ & $28.1 \% 137 / 487$ & \\
\hline $\mathrm{SBP}[\mathrm{mmHg}]$ & $130(114-150) ; n=745$ & $130(110-140) ; n=255$ & $131(120-150) ; n=490$ & 0.002 \\
\hline $\mathrm{DBP}[\mathrm{mmHg}]$ & $80(70-90) ; n=745$ & $80(70-84) ; n=255$ & $80(70-90) ; n=490$ & 0.0005 \\
\hline $\mathrm{HR}[\mathrm{bpm}]$ & $80(70-90) ; \mathrm{n}=747$ & $70(60-75) ; n=256$ & $84(75-100) ; n=491$ & $<0.0001$ \\
\hline QRS duration [ms] & $102(91-120) ; n=673$ & $102(92-121) ; n=227$ & $102(90-120) ; n=446$ & 0.67 \\
\hline ACS as a cause of admission & $25.2 \% ; 188 / 746$ & $20.7 \% ; 53 / 256$ & $27.5 \% ; 135 / 490$ & 0.04 \\
\hline \multicolumn{5}{|c|}{ Laboratory findings at admission } \\
\hline Serum sodium [mmol/L] & $\begin{array}{c}139.0(136.0-141.0) \\
n=738\end{array}$ & $\begin{array}{c}139.0(136.0-141.0) \\
n=252\end{array}$ & $\begin{array}{c}139.0(136.6-141.0) \\
n=486\end{array}$ & 0.39 \\
\hline Serum potassium $[\mathrm{mmol} / \mathrm{L}]$ & $\begin{array}{c}4.4(4.1-4.8) \\
n=738\end{array}$ & $\begin{array}{c}4.49(4.12-4.83) \\
n=252\end{array}$ & $\begin{array}{c}4.40(4.06-4.76) \\
n=486\end{array}$ & 0.06 \\
\hline Serum creatinine $[\mathrm{mg} / \mathrm{dL}]$ & $\begin{array}{c}1.05(0.87-1.32) \\
n=725\end{array}$ & $\begin{array}{c}1.01(0.85-1.30) \\
n=248\end{array}$ & $\begin{array}{c}1.07(0.89-1.33) \\
n=477\end{array}$ & 0.11 \\
\hline Hemoglobin $[\mathrm{g} / \mathrm{dL}]$ & $\begin{array}{c}13.4(12.3-14.6) \\
n=734\end{array}$ & $\begin{array}{c}13.4(12.1-14.7) \\
\mathrm{n}=251\end{array}$ & $\begin{array}{c}13.4(12.4-14.6) \\
n=483\end{array}$ & 0.61 \\
\hline
\end{tabular}


Table 1 (cont.). Baseline characteristics, clinical course of index hospitalization, in-hospital and long-term outcomes of the reduced HR and not-reduced HR groups.

\begin{tabular}{|c|c|c|c|c|}
\hline & $\begin{array}{c}\text { Total } \\
(\mathrm{n}=747)\end{array}$ & $\begin{array}{l}\text { Not-reduced HR } \\
\quad(n=256)\end{array}$ & $\begin{array}{l}\text { Reduced HR } \\
(n=491)\end{array}$ & $\mathbf{P}$ \\
\hline \multicolumn{5}{|c|}{ Major management and pharmacotherapy during index hospitalization, clinical status at discharge } \\
\hline $\begin{array}{l}\mathrm{PCI} / \mathrm{CABG} \text { during } \\
\text { hospitalization }\end{array}$ & $16.8 \% ; 125 / 745$ & $16.1 \% ; 41 / 254$ & $17.1 \% ; 84 / 491$ & 0.76 \\
\hline Beta-blocker & $89.9 \%(670 / 745)$ & $87.4 \%(222 / 254)$ & $91.2 \%(448 / 491)$ & 0.12 \\
\hline Digoxin & $15.0 \%(112 / 745)$ & $14.1 \%(36 / 254)$ & $15.5 \%(76 / 491)$ & 0.67 \\
\hline Amiodarone & $10.6 \%(79 / 745)$ & $8.7 \%(22 / 254)$ & $11.6 \%(57 / 491)$ & 0.26 \\
\hline Antiarrhythmics & $4.0 \%(30 / 745)$ & $5.1 \%(13 / 254)$ & $3.5 \%(17 / 491)$ & 0.33 \\
\hline $\mathrm{HR}[\mathrm{bpm}]$ & $70(64-78) ; n=747$ & $72(68-80) ; n=256$ & $70(62-75) ; n=491$ & $<0.0001$ \\
\hline $\mathrm{SBP}[\mathrm{mmHg}]$ & $120(110-130) ; n=744$ & $120(110-130) ; n=255$ & $120(110-130) ; n=489$ & 0.91 \\
\hline $\mathrm{DBP}[\mathrm{mmHg}]$ & $70(65-80) ; n=742$ & $70(65-80) ; n=254$ & $70(65-80) ; n=488$ & 0.16 \\
\hline \multicolumn{5}{|c|}{ Pharmacotherapy at hospital discharge } \\
\hline Diuretics & $82.1 \% ; 613 / 747$ & $79.3 \% ; 203 / 256$ & $83.5 \% ; 410 / 491$ & 0.16 \\
\hline Aldosterone antagonist & $63.1 \% ; 471 / 746$ & $65.2 \% ; 167 / 256$ & $62.0 \% ; 304 / 490$ & 0.42 \\
\hline ACEI & $77.5 \% ; 579 / 747$ & $77.3 \% ; 198 / 256$ & $77.6 \% ; 381 / 491$ & 0.93 \\
\hline ARB & $10.6 \% ; 79 / 745$ & $9.2 \% ; 23 / 255$ & $11.4 \% ; 56 / 490$ & 0.38 \\
\hline Beta-blocker & $89.3 \% ; 667 / 747$ & $87.1 \% ; 223 / 256$ & $90.4 \% ; 444 / 491$ & 0.17 \\
\hline Statins & $74.7 \% ; 558 / 747$ & $73.4 \% ; 188 / 256$ & $75.4 \% ; 370 / 491$ & 0.60 \\
\hline Antiplatelets & $78.9 \% ; 589 / 747$ & $78.9 \% ; 202 / 256$ & $78.8 \% ; 387 / 491$ & 1.00 \\
\hline Ivabradine & $0.5 \% ; 2 / 391$ & $0.0 \% ; 0 / 145$ & $0.8 ; 2 / 246$ & 0.53 \\
\hline \multicolumn{5}{|l|}{ In-hospital outcome } \\
\hline $\begin{array}{l}\text { Hospitalization length } \\
\text { [days] }\end{array}$ & $7(4-10) ; n=722$ & $6(3-9) ; n=246$ & $7(4-11) ; n=476$ & 0.004 \\
\hline \multicolumn{5}{|l|}{ One-year outcome } \\
\hline One-year all-cause death & $11.6 \% ; 84 / 722$ & $10.5 \% ; 26 / 246$ & $12.2 \% ; 58 / 476$ & 0.54 \\
\hline $\begin{array}{l}\text { One-year all-cause death } \\
\text { or rehospitalization due to } \\
\text { the HF worsening }\end{array}$ & $29.8 \% ; 180 / 605$ & $27.1 \% ; 56 / 207$ & $31.2 \% ; 124 / 398$ & 0.30 \\
\hline
\end{tabular}

Bolded values indicate $p$-values < 0.05. ACEI - angiotensin converting enzyme inhibitor; ACS - acute coronary syndrome; ARB - angiotensin receptor blocker; $\mathrm{BMI}$ — body mass index; CABG — coronary artery bypass grafting; COPD - chronic obstructive pulmonary disease; $\mathrm{DBP}$ - diastolic blood pressure; HF — heart failure; HR — heart rate; LVEF — left ventricular ejection fraction; NYHA — New York Heart Association; $\mathrm{PCl}$ - percutaneous coronary intervention; SBP — systolic blood pressure

Table 2. Comparison of patients enrolled in the ESC-HF-Pilot and ESC-HF-LT Registries in regard to heart rate $(\mathrm{HR})$ values.

\begin{tabular}{lccc}
\hline & ESC-HF-Pilot Registry & ESC-HF-LT Registry & $P$ \\
\hline HR at admission $[\mathrm{bpm}]$ & $80(70-95)$ & $78(68-90)$ & $\mathbf{0 . 0 2}$ \\
HR at discharge $[\mathrm{bpm}]$ & $70(65-78)$ & $70(62-77)$ & 0.16 \\
Median HR reduction during hospitalization $[\mathrm{bpm}]$ & $10(0-20)$ & $6(0-20)$ & 0.06 \\
Patients who achieved HR reduction & $68.9 \%$ & $62.9 \%$ & 0.09 \\
\hline
\end{tabular}

Bolded values indicates p-values < 0.05. ESC-HF-Pilot - European Society of Cardiology Heart Failure Pilot; ESC-HF-LT — European Society of Cardiology Long-Term

admission in comparison to the not-reduced HR group, however this observation did not reach the statistical significance. Moreover, without signifi- cance, the analysis of in-hospital pharmacotherapy showed a higher percentage of patients receiving $\mathrm{BBs}$ in the reduced HR group. At discharge, the 


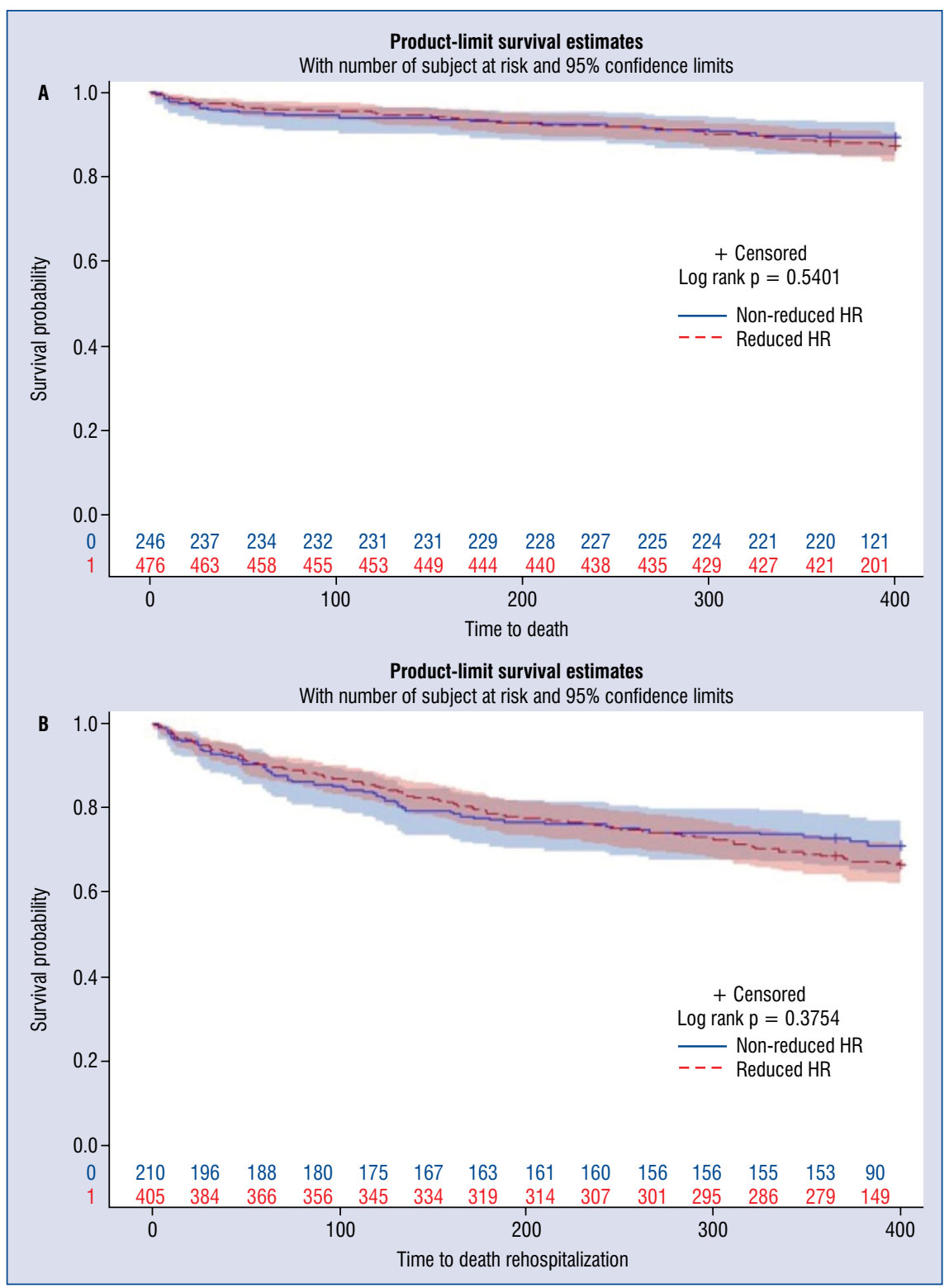

Figure 2. Kaplan-Meier curves in the reduced HR and not-reduced HR groups; A. For all-cause 12-month mortality; B. For all-cause 12-month mortality or hospitalization; HR - heart rate.

reduced HR group more often had been prescribed BBs. A lower percentage of patients receiving BBs during index hospitalization and at discharge may, at least partially, result from a higher occurrence of chronic obstructive pulmonary disease in this group.

Additionally, in the HR reduction group a higher percentage of patients presented with ACS as a cause of admission. Myftiu et al. [16] reported that, in patients presenting with acute myocardial infarction (AMI) the group with $\mathrm{HF}$ upon admission had significantly higher HR at admission in comparison to the AMI without HF group. Moreover, myocardial infarction may be a reason for $\mathrm{BB}$ implementation, which contributes to a reduction of $\mathrm{HR}$.

Several recent clinical trials and populationbased studies reported significant associations between HR and outcomes in patients with HF. Previous analysis of the ESC-HF Pilot Registry showed that higher HR at admission was associated with worse clinical course during index hospitalization [5]. The placebo-subgroup analysis of patients with stable coronary artery disease and left-ventricular dysfunction enrolled in the BEAUTIFUL (morBidity - mortality EvAlUaTion of the 
Table 3. Univariate analysis of predictors of primary and secondary endpoints at 1 year in the reduced heart rate (HR) group.

\begin{tabular}{|c|c|c|c|c|}
\hline & \multicolumn{2}{|c|}{ Primary endpoint } & \multicolumn{2}{|c|}{ Secondary endpoint } \\
\hline & Hazard ratio $(95 \% \mathrm{Cl})$ & $\mathbf{P}$ & Hazard ratio $(95 \% \mathrm{Cl})$ & $\mathbf{P}$ \\
\hline \multicolumn{5}{|l|}{ Demographics } \\
\hline Age, per 10 years & $1.71(1.34-2.17)$ & $<0.0001$ & $1.12(0.97-1.28)$ & 0.12 \\
\hline Male & $0.77(0.45-1.29)$ & 0.32 & $0.81(0.57-1.16)$ & 0.26 \\
\hline $\mathrm{BMI}$, per $1 \mathrm{~kg} / \mathrm{m}^{2}$ & $0.93(0.88-0.98)$ & 0.01 & $0.99(0.96-1.02)$ & 0.63 \\
\hline \multicolumn{5}{|l|}{ Heart failure } \\
\hline LVEF, per $5 \%$ & $0.98(0.89-1.08)$ & 0.67 & $0.83(0.74-0.94)$ & 0.004 \\
\hline \multicolumn{5}{|l|}{ Medical history } \\
\hline Hypertension & $1.04(0.58-1.85)$ & 0.90 & $0.77(0.53-1.12)$ & 0.17 \\
\hline Coronary artery disease & $0.99(0.58-1.67)$ & 0.96 & $1.30(0.91-1.86)$ & 0.15 \\
\hline Peripheral artery disease & $1.76(0.92-3.40)$ & 0.09 & $1.27(0.77-2.09)$ & 0.35 \\
\hline Diabetes & $1.41(0.83-2.37)$ & 0.20 & $1.43(1.01-2.03)$ & 0.04 \\
\hline Chronic kidney disease & $2.02(1.16-3.52)$ & 0.01 & $1.78(1.22-2.60)$ & 0.003 \\
\hline COPD & $1.29(0.70-2.39)$ & 0.42 & $1.33(0.89-2.00)$ & 0.17 \\
\hline Stroke & $0.94(0.37-2.34)$ & 0.89 & $1.91(1.16-3.14)$ & 0.01 \\
\hline \multicolumn{5}{|l|}{ Clinical status at admission } \\
\hline NYHA class, per 1 class & $2.09(1.44-3.04)$ & 0.0001 & $1.66(1.32-2.10)$ & $<0.0001$ \\
\hline $\mathrm{SBP}$, per $10 \mathrm{mmHg}$ & $0.95(0.87-1.04)$ & 0.28 & $0.89(0.84-0.95)$ & 0.0004 \\
\hline $\mathrm{DBP}$, per $10 \mathrm{mmHg}$ & $0.97(0.82-1.14)$ & 0.68 & $0.90(0.81-1.01)$ & 0.07 \\
\hline $\mathrm{HR}$, per $10 \mathrm{bpm}$ & $1.10(0.98-1.23)$ & 0.11 & $1.05(0.97-1.13)$ & 0.25 \\
\hline QRS reduction, per $10 \mathrm{~ms}$ & $1.06(0.96-1.18)$ & 0.25 & $1.05(0.98-1.12)$ & 0.20 \\
\hline Cardiogenic shock & $1.53(0.37-6.27)$ & 0.56 & $1.36(0.50-3.67)$ & 0.55 \\
\hline VF or VT as a cause of admission & $0.96(0.35-2.65)$ & 0.94 & $0.90(0.46-1.76)$ & 0.75 \\
\hline ACS as a cause of admission & $1.30(0.75-2.26)$ & 0.32 & $1.06(0.72-1.58)$ & 0.77 \\
\hline \multicolumn{5}{|l|}{ Laboratory findings at admission } \\
\hline Serum sodium, per $1 \mathrm{mmol} / \mathrm{L}$ & $0.89(0.85-0.94)$ & $<0.0001$ & $0.94(0.90-0.97)$ & 0.001 \\
\hline Serum potassium, per $1 \mathrm{mmol} / \mathrm{L}$ & $0.90(0.57-1.42)$ & 0.64 & $0.83(0.61-1.14)$ & 0.25 \\
\hline Serum creatinine, per $1 \mathrm{mg} / \mathrm{dL}$ & $1.27(0.94-1.72)$ & 0.13 & $1.28(1.03-1.59)$ & 0.02 \\
\hline Hemoglobin, per $1 \mathrm{~g} / \mathrm{dL}$ & $0.83(0.72-0.94)$ & 0.004 & $0.88(0.81-0.97)$ & 0.01 \\
\hline \multicolumn{5}{|c|}{$\begin{array}{l}\text { Major management during index hospitalization, } \\
\text { clinical status and laboratory findings at discharge }\end{array}$} \\
\hline PCI/CABG during hospitalization & $0.84(0.41-1.82)$ & 0.70 & $1.04(0.63-1.71)$ & 0.88 \\
\hline HR, per 10 bpm & $1.31(1.02-1.68)$ & 0.03 & $1.15(0.97-1.37)$ & 0.10 \\
\hline $\mathrm{SBP}$, per $10 \mathrm{mmHg}$ & $0.72(0.60-0.85)$ & 0.0001 & $0.78(0.70-0.88)$ & $<0.0001$ \\
\hline $\mathrm{DBP}$, per $10 \mathrm{mmHg}$ & $0.70(0.54-0.91)$ & 0.008 & $0.74(0.62-0.88)$ & 0.0006 \\
\hline \multicolumn{5}{|c|}{ Pharmacotherapy at hospital discharge } \\
\hline Diuretics & $1.31(0.62-2.75)$ & 0.48 & $1.44(0.88-2.72)$ & 0.15 \\
\hline Aldosterone antagonist & $0.84(0.50-1.42)$ & 0.52 & $1.24(0.87-1.77)$ & 0.24 \\
\hline ACEI & $0.60(0.34-1.03)$ & 0.06 & $0.69(0.48-1.01)$ & 0.05 \\
\hline ARB & $0.73(0.29-1.82)$ & 0.50 & $1.12(0.66-1.89)$ & 0.70 \\
\hline Beta-blocker & $0.47(0.24-0.91)$ & 0.02 & $0.82(0.49-1.38)$ & 0.45 \\
\hline \multicolumn{5}{|c|}{ Pharmacotherapy prior hospital admission } \\
\hline Diuretics & $1.27(0.73-2.21)$ & 0.40 & $1.66(1.13-2.42)$ & 0.009 \\
\hline Aldosteron antagonist & $0.84(0.48-1.45)$ & 0.52 & $1.13(0.79-1.60)$ & 0.51 \\
\hline ACEI & $1.35(0.77-2.37)$ & 0.29 & $1.03(0.72-1.47)$ & 0.89 \\
\hline ARB & $1.00(0.43-2.34)$ & 0.99 & $1.07(0.62-1.83)$ & 0.81 \\
\hline Beta-blocker & $0.91(0.52-1.61)$ & 0.75 & $1.07(0.72-1.60)$ & 0.72 \\
\hline Statins & $0.65(0.38-1.10)$ & 0.11 & $1.09(0.77-1.56)$ & 0.62 \\
\hline Antiplatelets & $1.19(0.69-2.07)$ & 0.54 & $1.22(0.85-1.76)$ & 0.28 \\
\hline
\end{tabular}

Bolded values indicate $\mathrm{p}$-values $<0.05$. ACEI — angiotensin converting enzyme inhibitor; ACS - acute coronary syndrome; ARB - angiotensin receptor blocker; $\mathrm{BMI}$ - body mass index; CABG - coronary artery bypass grafting; $\mathrm{Cl}$ - confidence interval; COPD - chronic obstructive pulmonary disease; DBP — diastolic blood pressure; LVEF — left ventricular ejection fraction; NYHA — New York Heart Association;

$\mathrm{PCl}$ - percutaneous coronary intervention; SBP — systolic blood pressure; VF — ventricular fibrillation; VT — ventricular tachycardia 
Table 4. Univariate analysis of predictors of primary and secondary endpoints at 1 year in the not-reduced heart rate (HR) group.

\begin{tabular}{|c|c|c|c|c|}
\hline & \multicolumn{2}{|l|}{ Primary endpoint } & \multicolumn{2}{|c|}{ Secondary endpoint } \\
\hline & Hazard ratio (95\% CI) & $\mathbf{P}$ & Hazard ratio (95\% CI) & $\mathbf{P}$ \\
\hline \multicolumn{5}{|l|}{ Demographics } \\
\hline Age, per 10 years & $1.46(1.05-2.02)$ & 0.02 & $1.13(0.92-1.38)$ & 0.24 \\
\hline Male & $0.81(0.36-1.82)$ & 0.61 & $0.88(0.51-1.51)$ & 0.63 \\
\hline $\mathrm{BMI}$, per $1 \mathrm{~kg} / \mathrm{m}^{2}$ & $0.95(0.87-1.04)$ & 0.24 & $0.97(0.92-1.02)$ & 0.24 \\
\hline \multicolumn{5}{|l|}{ Heart failure } \\
\hline LVEF, per $5 \%$ & $0.78(0.56-1.07)$ & 0.12 & $0.80(0.65-0.98)$ & 0.03 \\
\hline \multicolumn{5}{|l|}{ Medical history } \\
\hline Hypertension & $1.66(0.70-3.96)$ & 0.25 & $0.84(0.50-1.41)$ & 0.50 \\
\hline Coronary artery disease & $2.35(0.89-6.23)$ & 0.09 & $2.45(1.27-4.72)$ & 0.01 \\
\hline Peripheral artery disease & $1.57(0.54-4.56)$ & 0.41 & $1.33(0.63-2.80)$ & 0.45 \\
\hline Diabetes & $1.81(0.84-3.92)$ & 0.14 & $1.06(0.61-1.82)$ & 0.84 \\
\hline Chronic kidney disease & $1.97(0.83-4.69)$ & 0.13 & $1.85(1.03-3.32)$ & 0.04 \\
\hline COPD & $2.10(0.84-5.23)$ & 0.11 & $1.47(0.74-2.89)$ & 0.27 \\
\hline Stroke & $0.00(0.00 \rightarrow 999)$ & 0.99 & $0.97(0.30-3.10)$ & 0.96 \\
\hline \multicolumn{5}{|l|}{ Clinical status at admission } \\
\hline NYHA class, per 1 class & $1.93(1.13-3.31)$ & 0.02 & $1.38(0.97-1.94)$ & 0.07 \\
\hline $\mathrm{SBP}$, per $10 \mathrm{mmHg}$ & $0.98(0.84-1.13)$ & 0.75 & $0.95(0.85-1.06)$ & 0.36 \\
\hline $\mathrm{DBP}$, per $10 \mathrm{mmHg}$ & $0.69(0.52-0.91)$ & 0.009 & $0.87(0.72-1.06)$ & 0.17 \\
\hline HR, per $10 \mathrm{bpm}$ & $0.96(0.68-1.37)$ & 0.83 & $1.30(1.03-1.64)$ & 0.03 \\
\hline QRS duration, per $10 \mathrm{~ms}$ & $1.08(0.96-1.22)$ & 0.18 & $1.04(0.94-1.14)$ & 0.42 \\
\hline Cardiogenic shock & $0.00(0.00 \rightarrow 999)$ & 0.99 & $0.00(0.00 \rightarrow 999)$ & 0.99 \\
\hline VF or VT as a cause of admission & $0.30(0.04-2.18)$ & 0.23 & $0.61(0.25-1.53)$ & 0.30 \\
\hline ACS as a cause of admission & $0.63(0.22-1.83)$ & 0.40 & $0.88(0.46-1.69)$ & 0.70 \\
\hline \multicolumn{5}{|l|}{ Laboratory findings at admission } \\
\hline Serum sodium, per $1 \mathrm{mmol} / \mathrm{L}$ & $0.90(0.82-0.99)$ & 0.03 & $0.90(0.84-0.97)$ & 0.003 \\
\hline Serum potassium, per $1 \mathrm{mmol} / \mathrm{L}$ & $1.52(0.85-2.72)$ & 0.15 & $1.19(0.77-1.83)$ & 0.43 \\
\hline Serum creatinine, per $1 \mathrm{mg} / \mathrm{dL}$ & $1.89(1.27-2.80)$ & 0.002 & $1.42(1.03-1.97)$ & 0.04 \\
\hline Hemoglobin, per $1 \mathrm{~g} / \mathrm{dL}$ & $0.84(0.72-0.99)$ & 0.04 & $0.89(0.79-0.996)$ & 0.04 \\
\hline \multicolumn{5}{|c|}{$\begin{array}{l}\text { Major management during index hospitalization, } \\
\text { clinical status and laboratory findings at discharge }\end{array}$} \\
\hline PCI/CABG during hospitalization & $0.44(0.10-0.87)$ & 0.27 & $0.67(0.30-1.48)$ & 0.32 \\
\hline $\mathrm{HR}$, per $10 \mathrm{bpm}$ & $1.05(0.87-1.28)$ & 0.59 & $1.06(0.95-1.19)$ & 0.31 \\
\hline $\mathrm{SBP}$, per $10 \mathrm{mmHg}$ & $0.79(0.62-1.00)$ & 0.053 & $0.80(0.68-0.93)$ & 0.005 \\
\hline DBP, per $10 \mathrm{mmHg}$ & $0.56(0.42-0.82)$ & 0.0015 & $0.97(0.57-0.94)$ & 0.016 \\
\hline \multicolumn{5}{|c|}{ Pharmacotherapy at hospital admission } \\
\hline Diuretics & $2.31(0.87-6.12)$ & 0.09 & $2.61(1.36-5.03)$ & 0.004 \\
\hline Aldosterone antagonist & $1.16(0.53-2.55)$ & 0.71 & $1.82(1.08-3.06)$ & 0.02 \\
\hline ACEI & $0.97(0.43-2.21)$ & 0.95 & $0.96(0.56-1.65)$ & 0.89 \\
\hline ARB & $0.46(0.06-3.39)$ & 0.44 & $0.95(0.38-2.38)$ & 0.92 \\
\hline Beta-blocker & $1.36(0.51-3.62)$ & 0.54 & $1.28(0.68-2.41)$ & 0.45 \\
\hline \multicolumn{5}{|c|}{ Pharmacotherapy prior hospital discharge } \\
\hline Diuretics & $0.93(0.37-2.32)$ & 0.88 & $1.22(0.63-2.35)$ & 0.55 \\
\hline Aldosterone antagonist & $1.21(0.32-2.78)$ & 0.65 & $1.59(0.89-2.86)$ & 0.12 \\
\hline ACEI & $0.38(0.18-0.84)$ & 0.02 & $0.42(0.25-0.72)$ & 0.001 \\
\hline ARB & $0.40(0.06-2.97)$ & 0.37 & $0.82(0.33-2.04)$ & 0.67 \\
\hline Beta-blocker & $0.68(0.26-1.81)$ & 0.44 & $0.71(0.37-1.38)$ & 0.31 \\
\hline Statins & $0.87(0.38-1.99)$ & 0.74 & $1.04(0.58-1.84)$ & 0.90 \\
\hline Antiplatelets & $0.75(0.31-1.78)$ & 0.51 & $1.04(0.56-1.92)$ & 0.91 \\
\hline
\end{tabular}

Bolded values indicate $p$-values $<0.05$. ACEI - angiotensin converting enzyme inhibitor; ACS - acute coronary syndrome; ARB - angiotensin receptor blocker; BMI — body mass index; CABG — coronary artery bypass grafting; COPD - chronic obstructive pulmonary disease; $\mathrm{DBP}$ - diastolic blood pressure; LVEF — left ventricular ejection fraction; NYHA - New York Heart Association; PCI - percutaneous coronary intervention; SBP — systolic blood pressure; VF — ventricular fibrillation; VT — ventricular tachycardia 
Table 5. Multivariate analysis of predictors of primary and secondary endpoints at 1 year in the reduced heart rate group.

\begin{tabular}{|c|c|c|c|c|c|}
\hline Primary endpoint & HR $(95 \% \mathrm{Cl})$ & $\mathbf{P}$ & Secondary endpoint & HR (95\% Cl) & $\mathbf{P}$ \\
\hline Age, per 10 years & $1.58(1.22-2.07)$ & $<0.001$ & LVEF, per $5 \%$ & $0.96(0.90-1.02)$ & 0.209 \\
\hline BMI, per 1 kg/m² & $0.96(0.91-1.02)$ & 0.217 & Diabetes & $1.40(0.96-2.05)$ & 0.080 \\
\hline Chronic kidney disease & $1.44(0.74-2.81)$ & 0.280 & Chronic kidney disease & $1.34(0.85-2.10)$ & 0.206 \\
\hline NYHA class at admission & 1.66 (1.09-2.54) & 0.019 & Stroke & $1.62(0.92-2.85)$ & 0.096 \\
\hline $\begin{array}{l}\text { Serum sodium at admission, } \\
\text { per } 1 \mathrm{mmol} / \mathrm{dL}\end{array}$ & $0.91(0.86-0.97)$ & 0.003 & $\begin{array}{l}\text { NYHA class } \\
\text { at admission }\end{array}$ & $1.29(0.98-1.68)$ & 0.065 \\
\hline $\begin{array}{l}\text { Hemoglobin at admission, } \\
\text { per } 1 \mathrm{~g} / \mathrm{dL}\end{array}$ & $0.98(0.82-1.16)$ & 0.790 & $\begin{array}{l}\text { SBP at admission, } \\
\text { per } 10 \mathrm{mmHg}\end{array}$ & $0.89(0.76-1.04)$ & 0.297 \\
\hline $\begin{array}{l}\text { Heart rate at discharge, } \\
\text { per } 10 \mathrm{bpm}\end{array}$ & $0.98(0.72-1.33)$ & 0.886 & $\begin{array}{l}\text { Serum sodium } \\
\text { at admission, per } 1 \mathrm{mmol} / \mathrm{dL}\end{array}$ & $0.96(0.92-1.00)$ & 0.058 \\
\hline $\begin{array}{l}\text { SBP at discharge, } \\
\text { per } 10 \mathrm{mmHg}\end{array}$ & $0.67(0.51-0.87)$ & 0.003 & $\begin{array}{l}\text { Serum creatinine } \\
\text { at admission, per } 1 \mathrm{mg} / \mathrm{dl}\end{array}$ & $1.07(0.77-1.49)$ & 0.688 \\
\hline $\begin{array}{l}\text { DBP at discharge, } \\
\text { per } 10 \mathrm{mmHg}\end{array}$ & $1.27(0.85-1.89)$ & 0.242 & $\begin{array}{l}\text { Hemoglobin } \\
\text { at admission, per } 1 \mathrm{~g} / \mathrm{dL}\end{array}$ & $0.99(0.85-1.03)$ & 0.188 \\
\hline $\begin{array}{l}\text { Beta-blocker } \\
\text { at discharge }\end{array}$ & $0.84(0.35-2.01)$ & 0.697 & $\begin{array}{l}\text { SBP at discharge, } \\
\text { per } 10 \mathrm{mmHg}\end{array}$ & $0.89(0.76-1.04)$ & 0.140 \\
\hline \multirow[t]{2}{*}{ Statins at discharge } & $0.52(0.26-1.02)$ & 0.057 & $\begin{array}{l}\text { DBP at discharge, } \\
\text { per } 10 \mathrm{mmHg}\end{array}$ & $1.03(0.81-1.31)$ & 0.827 \\
\hline & & & Prior diuretics usage & $1.23(0.82-1.87)$ & 0.320 \\
\hline
\end{tabular}

Bolded values indicates p-values < 0.05. BMI — body mass index; Cl — confidence interval; DBP — diastolic blood pressure; HR - hazard ratio; LVEF — left ventricular ejection fraction; NYHA — New York Heart Association; SBP — systolic blood pressure

Table 6. Multivariate analysis of predictors of primary and secondary endpoints at 1 year in the not-reduced heart rate group.

\begin{tabular}{|c|c|c|c|c|c|}
\hline Primary endpoint & HR (95\% Cl) & $\mathbf{P}$ & Secondary endpoint & HR (95\% Cl) & $\mathbf{P}$ \\
\hline Age, per 10 years & $1.25(0.88-1.78)$ & 0.213 & LVEF, per $5 \%$ & $0.95(0.83-1.08)$ & 0.422 \\
\hline NYHA class at admission & $1.73(0.93-3.21)$ & 0.082 & Coronary artery disease & $2.13(0.92-4.93)$ & 0.078 \\
\hline $\mathrm{DBP}$ at admission, per $10 \mathrm{mmHg}$ & $0.90(0.69-1.18)$ & 0.432 & Chronic kidney disease & $1.38(0.68-2.83)$ & 0.377 \\
\hline $\begin{array}{l}\text { Serum sodium at admission, } \\
\text { per } 1 \mathrm{mmol} / \mathrm{dL}\end{array}$ & $0.96(0.85-1.07)$ & 0.434 & $\begin{array}{l}\text { Serum sodium at } \\
\text { admission, per } 1 \mathrm{mmol} / \mathrm{dL}\end{array}$ & $0.95(0.86-1.04)$ & 0.259 \\
\hline $\begin{array}{l}\text { Serum creatinine at admission, } \\
\text { per } 1 \mathrm{mg} / \mathrm{dL}\end{array}$ & $1.62(0.98-2.70)$ & 0.061 & $\begin{array}{l}\text { Serum creatinine at } \\
\text { admission, per } 1 \mathrm{mg} / \mathrm{dL}\end{array}$ & $1.02(0.59-1.77)$ & 0.942 \\
\hline $\begin{array}{l}\text { Hemoglobin at admission, } \\
\text { per } 1 \mathrm{~g} / \mathrm{dL}\end{array}$ & $0.93(0.74-1.17)$ & 0.543 & $\begin{array}{l}\text { Hemoglobin at admission, } \\
\text { per } 1 \mathrm{~g} / \mathrm{dL}\end{array}$ & $0.97(0.82-1.14)$ & 0.684 \\
\hline DBP at discharge, per $10 \mathrm{mmHg}$ & $0.64(0.43-0.95)$ & 0.026 & SBP at discharge, & $0.90(0.68-1.19)$ & 0.441 \\
\hline \multirow{5}{*}{ ACEl at discharge } & \multirow[t]{5}{*}{$0.79(0.30-2.04)$} & \multirow[t]{5}{*}{0.619} & & & \\
\hline & & & $\begin{array}{l}\text { DBP at discharge, } \\
\text { per } 10 \mathrm{mmHg}\end{array}$ & $1.09(0.70-1.69)$ & 0.705 \\
\hline & & & $\begin{array}{l}\text { Prior aldosterone } \\
\text { antagonist usage }\end{array}$ & $1.22(0.60-2.49)$ & 0.584 \\
\hline & & & Prior diuretics usage & $1.99(0.84-4.72)$ & 0.118 \\
\hline & & & ACEI at discharge & $0.48(0.23-0.99)$ & 0.047 \\
\hline
\end{tabular}

Bolded text indicates p-values < 0.05. ACEI — angiotensin converting enzyme inhibitor; $\mathrm{BMI}$ - body mass index; $\mathrm{Cl}$ - confidence interval; DBP — diastolic blood pressure; HR - hazard ratio; LVEF — left ventricular ejection fraction; NYHA — New York Heart Association;

SBP — systolic blood pressure 
$I_{\mathrm{f}}$ inhibitor ivabradine in patients with coronary disease and left ventricULar dysfunction) study revealed, that a baseline resting $\mathrm{HR} \geq 70 \mathrm{bpm}$ in comparison to $\mathrm{HR}<70 \mathrm{bpm}$ is associated with a significantly higher risk of several outcomes, including cardiovascular death, admission to hospital for $\mathrm{HF}$, admission to hospital for myocardial infarction and coronary revascularization [9]. Moreover, in the SHIFT (Systolic Heart failure treatment with the $I_{\mathrm{f}}$ inhibitor ivabradine Trial) trial conducted on patients with chronic $\mathrm{HF}$, the placebo-treated group with $H R$ values $\geq 87 \mathrm{bpm}$ had significantly higher risk for the primary composite endpoint (cardiovascular death or hospital admission for worsening $\mathrm{HF}$ ) in comparison to the placebo-treated patients with HR from 70 to $72 \mathrm{bpm}$ [17]. In the ivabradinetreated group patients with $\mathrm{HR}<60 \mathrm{bpm}$ at 28 days of treatment the primary composite endpoint occurred less frequently during the observation in comparison to the group of patients with higher values of HR and the observed effect of ivabradine was shown to be $\mathrm{HR}$ reduction-dependent [17]. The ESC-HF-Pilot and ESC-HF-LT Registries did not include information concerning the in-hospital use of ivabradine.. The analysis of hospitalized $\mathrm{HF}$ with reduced ejection fraction (HFrEF) patients enrolled in the EVEREST (Efficacy of Vasopressin Antagonism in Heart Failure: Outcome Study With Tolvaptan) trial showed, that baseline HR was not associated with all-cause mortality. However at the level of $\geq 70 \mathrm{bpm}$, each 5-beat increase observed at 1 and 4 weeks following discharge was a predictor of all-cause mortality [18]. The study conducted by Kapoor et al. [19] enrolled patients with $\mathrm{HF}$ with preserved ejection fraction (HFpEF) revealed that all-cause mortality at one year is significantly higher in patients with $\mathrm{HR} \geq 60 \mathrm{bpm}$ or more in comparison to the group with $\mathrm{HR}<60 \mathrm{bpm}$. An interestingly high prevalence of digoxin usage was observed in both subgroups in the present analysis, however no difference between subgroups was observed. It is worth noting, that patients with paroxysmal atrial fibrillation were not excluded from the analysis and overall frequency of digoxin usage during the first years of data gathering was higher.

Analysis performed by Bui et al. [20] of HF hospitalized patients enrolled in the Get With The Guidelines-Hart Failure program showed a J-shaped correlation of in-hospital mortality and HR, whereas the lowest mortality rate was observed within $\mathrm{HR}$ values between $70 \mathrm{bpm}$ and $75 \mathrm{bpm}$, moreover, higher HR at admission is independently associated with higher in-hospital mortality [20].
The analysis of the Acute Decompensated Heart Failure Syndromes [21] Registry revealed, that in patients hospitalized for acute HF lower baseline $\mathrm{HR}$ is associated with a significantly higher rate of in-hospital cardiac death [22]. Moreover, Lancellotti et al. [23] reported, that increased HR at 24-36 h following admission for acute HF is related to a higher risk of in-hospital mortality. The impact of higher HR at discharge on poor prognosis of $\mathrm{HF}$ patients has also been reported [24]. Habal et al. [24] analyzed a group of discharged HF patients and revealed a significant increase in all-cause 1-month mortality for the value of discharge $\mathrm{HR} \geq 81 \mathrm{bpm}$ in comparison to the control group with HR 61-70 bpm. Moreover, the group of patients with HR $>90 \mathrm{bpm}$ had significantly increased risk of one-year all-cause mortality when compared to the controls (HR 40-60 $\mathrm{bpm})$ and also had higher rate of $\mathrm{HF}$ readmissions and cardiovascular disease within 30 days [24].

Laskey et al. [12] reported, in patients with SR HR $\geq 75 \mathrm{bpm}$ at hospital discharge increased the risk of 1-month and 1-year mortality and composite outcome of mortality and all-cause rehospitalization. The data concerning the impact of HR reduction on the prognosis of HF patients remains controversial. The results of the BEAUTIFUL study revealed no significant difference in the primary composite endpoint (cardiovascular death, admission to hospital for AMI and admission to hospital for new-onset or worsening HF) between ivabradine- and placebo-treated group [25]. However, in the subgroup of patients with HR $\geq 70 \mathrm{bpm}$, treatment with ivabradine significantly reduced the occurrence of coronary endpoints admission to hospital for myocardial infarction (fatal and non-fatal), admission to hospital for myocardial infarction or unstable angina and coronary revascularization. In the present study only 1 patient from the HR reduction group was using ivabradine and this difference between the two analyzed groups of patients did not reach statistical significance. The Cardiac Insufficiency BIsoprolol Study II (CIBIS II) revealed, that the lowest baseline HR and greatest HR changed during 2 months following inclusion due to bisoprolol usage in HF patients significantly reducing 1-year mortality and $\mathrm{HF}$ admission rate [10]. Li et al. [26] reported, that in- and outpatients with HFrEF in SR, who were enrolled in the Swedish Heart Failure Registry, had significant relation of higher HR with increased mortality. BB use significantly reduced HR in comparison to nontreated group and was related to reduced mortality, however, treatment with BBs did not change the association between HR and all-cause mortality [26]. 
In the present analysis, differences in usage of $\mathrm{BB}$ were observed. Compared with the not-reduced HR group, in the HR reduced group fewer patients used BBs before admission and more of them used $\mathrm{BBs}$ at discharge from the hospital. However, these discrepancies did not reach statistical significance.

\section{Conclusions}

The current study evaluates the impact of in-hospital HR reduction during hospitalization in HF patients on 1-year mortality and rehospitalization. The results of the present study revealed that $\mathrm{HR}$ reduction during hospitalization for $\mathrm{HF}$ is not associated with outcome of patients with SR. Moreover, predictors of primary endpoint and secondary endpoint were similar in patients with and without HR reduction during index hospitalization.

\section{Conflict of interest: None declared}

\section{References}

1. Balsam P, Tymińska A, Kapłon-Cieślicka A, et al. Predictors of one-year outcome in patients hospitalised for heart failure: results from the Polish part of the Heart Failure Pilot Survey of the European Society of Cardiology. Kardiol Pol. 2016; 74(1): 9-17, doi: 10.5603/KP.a2015.0112, indexed in Pubmed:26101021.

2. Crespo-Leiro MG, Anker SD, Maggioni AP, et al. European Society of Cardiology Heart Failure Long-Term Registry (ESC-HFLT): 1-year follow-up outcomes and differences across regions. Eur J Heart Failure. 2016; 18: 613-625.

3. Ozierański K, Balsam P, Tymińska A, et al. Heart failure in elderly patients: differences in clinical characteristics and predictors of 1-year outcome in the Polish ESC-HF Long-Term Registry. Pol Arch Med Wewn. 2016; 126(7-8): 502-513, doi: 10.20452/ pamw.3490, indexed in Pubmed: 27510261.

4. Kapłon-Cieślicka A, Tymińska A, Peller M, et al. Diagnosis, Clinical Course, and 1-Year Outcome in Patients Hospitalized for Heart Failure With Preserved Ejection Fraction (from the Polish Cohort of the European Society of Cardiology Heart Failure Long-Term Registry). Am J Cardiol. 2016; 118(4): 535-542, doi: 10.1016/j.amjcard.2016.05.046, indexed in Pubmed: 27374606.

5. Kapłon-Cieślicka A, Balsam P, Ozierański K, et al. Resting heart rate at hospital admission and its relation to hospital outcome in patients with heart failure. Cardiol J. 2014; 21(4): 425-433, doi: 10.5603/CJ.a2013.0147, indexed in Pubmed: 24142684.

6. Targher G, Dauriz M, Laroche C, et al. ESC-HFA HF Long-Term Registry investigators. In-hospital and 1-year mortality associated with diabetes in patients with acute heart failure: results from the ESC-HFA Heart Failure Long-Term Registry. Eur J Heart Fail. 2017; 19(1): 54-65, doi: 10.1002/ejhf.679, indexed in Pubmed: 27790816.

7. Kannel WB, Kannel C, Paffenbarger RS, et al. Heart rate and cardiovascular mortality: the Framingham Study. Am Heart J. 1987; 113(6): 1489-1494, indexed in Pubmed: 3591616.

8. Kolloch R, Legler UF, Champion A, et al. Impact of resting heart rate on outcomes in hypertensive patients with coronary artery disease: findings from the INternational VErapamil-SR/trandolapril STudy (INVEST). Eur Heart J. 2008; 29(10): 1327-1334, doi: 10.1093/eurheartj/ehn123, indexed in Pubmed:18375982.

9. Fox K, Ford I, Steg PG, et al. Heart rate as a prognostic risk factor in patients with coronary artery disease and left-ventricular systolic dysfunction (BEAUTIFUL): a subgroup analysis of a randomised controlled trial. Lancet. 2008; 372(9641): 817-821, doi: 10.1016/S0140-6736(08)61171-X, indexed in Pubmed: 18757091.

10. Lechat P, Hulot JS, Escolano S, et al. Heart rate and cardiac rhythm relationships with bisoprolol benefit in chronic heart failure in CIBIS II Trial. Circulation. 2001; 103(10): 1428-1433, indexed in Pubmed: 11245648.

11. Pocock SJ, Wang D, Pfeffer MA, et al. Predictors of mortality and morbidity in patients with chronic heart failure. Eur Heart J. 2006; 27(1): 65-75, doi:10.1093/eurheartj/ehi555, indexed in Pubmed: 16219658.

12. Laskey WK, Alomari I, Cox M, et al. Heart rate at hospital discharge in patients with heart failure is associated with mortality and rehospitalization. J Am Heart Assoc. 2015; 4(4), doi: 10.1161/ JAHA.114.001626, indexed in Pubmed: 25904590.

13. Maggioni AP, Dahlström U, Filippatos G, et al. Heart Failure Association of the European Society of Cardiology (HFA), Heart Failure Association of ESC (HFA). EURObservational Research Programme: the Heart Failure Pilot Survey (ESC-HF Pilot). Eur J Heart Fail. 2010; 12(10): 1076-1084, doi:10.1093/eurjhf/hfq154, indexed in Pubmed: 20805094.

14. Maggioni AP, Dahlström U, Filippatos G, et al. EURObservational Research Programme: regional differences and 1-year followup results of the Heart Failure Pilot Survey (ESC-HF Pilot). Eur J Heart Fail. 2013; 15(7): 808-817, doi: 10.1093/eurjhf/hft050, indexed in Pubmed: 23537547.

15. Ahmed A, Aronow WS, Fleg JL. Higher New York Heart Association classes and increased mortality and hospitalization in patients with heart failure and preserved left ventricular function. Am Heart J. 2006; 151(2): 444-450, doi: 10.1016/j. ahj.2005.03.066, indexed in Pubmed: 16442912.

16. Myftiu S, Bara P, Sharka I, et al. Heart failure predictors in a group of patients with myocardial infarction. Open Access Maced J Med Sci. 2016; 4(3): 435-438, doi: 10.3889/oamjms.2016.101, indexed in Pubmed: 27703569.

17. Böhm M, Swedberg K, Komajda M, et al. Heart rate as a risk factor in chronic heart failure (SHIFT): the association between heart rate and outcomes in a randomised placebo-controlled trial. Lancet. 2010; 376(9744): 886-894, doi: 10.1016/S01406736(10)61259-7, indexed in Pubmed: 20801495.

18. Greene SJ, Vaduganathan M, Wilcox JE, et al. The prognostic significance of heart rate in patients hospitalized for heart failure with reduced ejection fraction in sinus rhythm: insights from the EVEREST (Efficacy of Vasopressin Antagonism in Heart Failure: Outcome Study With Tolvaptan) trial. JACC Heart Fail. 2013; 1(6): 488-496, doi: 10.1016/j.jchf.2013.08.005, indexed in Pubmed: 24622000.

19. Kapoor JR, Heidenreich PA. Heart rate predicts mortality in patients with heart failure and preserved systolic function. J Card Fail. 2010; 16(10): 806-811, doi: 10.1016/j.cardfail.2010.04.013, indexed in Pubmed: 20932462.

20. Bui AL, Grau-Sepulveda MV, Hernandez AF, et al. Admission heart rate and in-hospital outcomes in patients hospitalized for heart failure in sinus rhythm and in atrial fibrillation. Am Heart J. 2013; 165(4): 567-574.e6, doi: 10.1016/j.ahj.2013.01.007, indexed in Pubmed: 23537974. 
21. Sato N, Gheorghiade M, Kajimoto K, et al. ATTEND Investigators. Hyponatremia and in-hospital mortality in patients admitted for heart failure (from the ATTEND registry). Am J Cardiol. 2013; 111(7): 1019-1025, doi: 10.1016/j.amjcard.2012.12.019, indexed in Pubmed: 23312128.

22. Kajimoto K, Sato N, Keida T, et al. Low admission heart rate is a marker rather than a mediator of increased in-hospital mortality for patients with acute heart failure syndromes in sinus rhythm. Int J Cardiol. 2014; 171(1): 98-100, doi: 10.1016/j.ijcard.2013.11.087, indexed in Pubmed: 24342405.

23. Lancellotti P, Ancion A, Magne J, et al. Elevated heart rate at 24-36h after admission and in-hospital mortality in acute in non-arrhythmic heart failure. Int J Cardiol. 2015; 182: 426-430, doi: 10.1016/j.ijcard.2015.01.027, indexed in Pubmed: 25596471.
24. Habal MV, Liu PP, Austin PC, et al. Association of heart rate at hospital discharge with mortality and hospitalizations in patients with heart failure. Circ Heart Fail. 2014; 7(1): 12-20, doi: 10.1161/CIRCHEARTFAILURE.113.000429, indexed in Pubmed: 24297690.

25. Fox K, Ford I, Steg P, et al. Ivabradine for patients with stable coronary artery disease and left-ventricular systolic dysfunction (BEAUTIFUL): a randomised, double-blind, placebo-controlled trial. Lancet. 2008; 372(9641): 807-816, doi: 10.1016/s01406736(08)61170-8.

26. Li SJ, Sartipy U, Lund LH, et al. Prognostic Significance of Resting Heart Rate and Use of $\beta$-Blockers in Atrial Fibrillation and Sinus Rhythm in Patients With Heart Failure and Reduced Ejection Fraction: Findings From the Swedish Heart Failure Registry. Circ Heart Fail. 2015; 8(5): 871-879, doi:10.1161/CIRCHEARTFAILURE.115.002285, indexed in Pubmed: 26243796. 